\title{
LOSSES IN SUPERCONDUCTORS FOR THE ENERGY DOUBLER
}

\author{
J.H. Murphy and M.S. Walker \\ Westinghouse Research Laboratories \\ Pittsburgh, Pennsylvania 15235 \\ February 17, 1975
}

\begin{abstract}
Peak field losses were calculated for various types of multifilament conductors, contemplated for the energy doubler program exposed to fields ramped in 4 to 30 seconds. Among the conductors examined were monolithic copper matrix and mixed matrix conductors and solder filled cables. General conclusions regarding conductor design have been stated, and recommendations for the energy doubler application have been made on the basis of loss and stability arguments.
\end{abstract}




\section{TABLE OF CONTENTS}

Abstract.............................. i

I. Introduction............................. 1

II. Theory of Ramped Field Losses in Rectangular Multifilamentary Superconductors.................. 3

III. Stability Considerations..................... 6

IV. Averaging Losses over Dipole Colls................ 9

v. Parametric Analysis.......................... 14

VI. Loss Calculations for Example Conductors............. 25

VII. Conclusions and Recommendations................. 33

VIII. Summary of Conductor Recommendations............. 37

IX. $\quad$ References................................ 38 


\title{
LOSSES IN SUPERCONDUCTORS FOR THE ENERGY DOUBLER
}

\author{
J.H. Murphy and M.S. Walker \\ Westinghouse Research Laboratories \\ Pittsburgh, Pennsylvania 15235
}

\section{Introduction}

To extend the Fermi National Accelerator Laboratory's 200/500 $\mathrm{GeV}$ proton accelerator to between 500 and $1000 \mathrm{GeV}$, superconducting magnets can be installed in the present accelerator site. Present estimates of this energy doubler device indicate that approximately 800 dipole and 400 quadrupole windings must be constructed from superconducting wire enclosed by separate vacuum vessels and magnetically enhanced by room temperature iron.

Losses will be generated in the superconducting windings as a result of their operation in the changing field environment. The energy doubler system requires that the superconductor design nominally meet a loss requirement of 1 watt/meter length of coil based upon the cost estimates of the system and the refrigeration restraints. The coils will be constructed from two rectangular conductor configurations, namely sizes of $0.075^{\prime \prime} \times 0.150^{\prime \prime}$ and $0.050^{\prime \prime} \times 0.150^{\prime \prime}$. These conductors will be pulsed between $2 \mathrm{~T}$ and $4.5 \mathrm{~T}$ repeatedly where for $10 \%$ of the cycle the conductor is at $2 \mathrm{~T}$, for $40 \%$ of the cycle the conductor is linearly ramped from $2 \mathrm{~T}$ to $4.5 \mathrm{~T}$, and for $10 \%$ of the cycle the conductor is at $4.5 \mathrm{~T}$. 
The period of the cycle may range as low as 10 seconds to a nominal value of 30 seconds.

This report has been written to assess the loss behavior which can be anticipated for several superconductor types that have been proposed by the Fermilab for the energy doubler and some additional types that may be considered for this application and to indicate some stability considerations. The approach taken has been to (1) set up the theoretical basis for the analysis of losses in conductor types, * (2) make calculations on a set of example conductors which have been chosen to span the various conductor types that should be considered and to provide an illustration of the effectiveness of various qualitative designs and parameters in reducing losses, (3) draw conclusions regarding rules to follow for the design of conductors for the energy doubler, and (4) recommend several conductor types for the Fermilab application. The organization of this report reflects that sequence of considerations.

*The theoretical basis for all of the calculations rests heavily upon work performed with support from the Office of Naval Research. A detailed consideration of ramped field losses is provided in the SemiAnnual Report for ONR Contract \#N00014-73-C-0461 which is current being prepared. 


\section{Theory of Ramped Field Losses in Rectangular Multifilament}

\section{Superconductors (MKS Units)}

To derive losses in multifilament superconductors, one must develop a model which accurately describes the phenomena under investigation. Carr ${ }^{(1,2)}$ proposed that in order to calculate the eddy current losses one could treat the conductor as a continuum with anisotropic conductivities. Parallel to the filaments the conductivity, $\sigma_{i n}$, is assumed to be large in comparison with the conductivity, $\sigma_{1}$, transverse to the filaments.

These conductivities are given by an average over a small volume containing many filaments. The average transverse conductivity, $\sigma_{L}$, which is used in the loss expressions is a particularly difficult parameter to estimate in practical conductors. The choice of the model to be used for $\sigma_{\perp}$ clearly depends upon the details of the conductor configuration as well as the conductivities of the various components. For the conditions where the average electric field parallel to the filaments approaches zero and the mean free path in the matrix is short in comparison to the interfilament spacing, Carr ${ }^{(3)}$ has shown that $\sigma_{\perp}$ is proportional to the matrix conductivity $\sigma_{m}$ and takes the form

$$
\sigma_{\perp}=\left(\frac{1-\lambda}{1+\lambda}\right) \quad \sigma_{m}
$$

for large interface resistance between the filament and the matrix or 


$$
\sigma_{\perp}=\left(\frac{1+\lambda}{1-\lambda}\right) \quad \sigma_{\mathrm{m}}
$$

where the interface resistance is small. $\lambda$ is defined as the fraction of the highest conductivity material in the total matrix. If only two materials are involved, then $\lambda$ refers to the fraction of superconductor in the matrix. If a mixed matrix or stranded cable is involved, then $\lambda$ includes the fraction of high conductivity material in contact with the filaments and the fraction of superconductor in the matrix.

A close examination of the path which a filament traverses throughout a rectangular multifilament superconductor reveals that a filament follows an elliptical spiral. The eddy current losses under ramped fields or low frequency alternating fields can therefore be calculated using a continuum with anisotropic conductivities. (4) For a linearly ramped transverse magnetic field with a $\dot{B}=\dot{B}_{0}$, the eddy current loss per unit volume is approximately given by

$$
\frac{P}{V}=\frac{\sigma_{1} B_{o}^{2}}{4}\left\{a^{2} \sin ^{2} \theta_{k}+b^{2} \cos ^{2} \theta_{k}+\left(\frac{L}{\pi}\right)^{2}\left[\left(\frac{b}{a}\right)^{2} \cos ^{2} \theta_{k}+\left(\frac{a}{b}\right)^{2} \sin ^{2} \theta_{k}\right]\right\}
$$

where $2 a$ is the width of the major axis, and $2 b$ is the width of the minor axis and $\theta_{k}$ is the angle measured from the major axis to the magnetic field vector.

In cylindrical conductors for thin saturation layers, if at

worst

$$
B<\left(\frac{2 \pi}{L}\right)^{2}\left[1+\left(\frac{2 \pi R_{o}}{L}\right)^{2}\right]^{-1 / 2} \frac{\lambda_{c} R_{o}}{\sigma_{\perp}}\left[1-\left(\frac{I}{I_{c}}\right)^{1 / 2}\right]
$$


then the dominant hysteresis loss is a filamentary loss. A similar criterla applies for a rectangular conductor.

The average filamentary hysteresis loss per unit volume is

$$
\frac{P_{h}}{V}=\frac{2 \lambda \bar{j}_{c} d|\dot{B}|}{3^{\pi}}
$$

provided that the filaments are not carrying any transport current. This hysteresis loss is only applicable for field changes very large in comparison with the field necessary to fully penetrate the superconducting filament.

The full penetration filamentary hysteresis loss when the filaments are carrying the critical current is (7)

$$
\frac{P_{h}}{V}=\frac{\lambda \bar{j}_{c} d|\dot{B}|}{2}
$$

for a transversely applied linearly ramped magnetic field. In the region of the conductor where transport current is being carried, the filamentary hysteresis loss is increased by at worst a factor of $\frac{3 \pi}{4}$ and in the region where no transport current is carried, the filament hysteresis loss is unaffected by the transport current. 


\section{Stability}

The selection or design of a superconductor for the energy doubler will depend upon a consideration of both conductor loss and stability. The stability problem can be roughly divided into three parts, thermal stability, electrical, magnetic or flux jump stability, and mechanical stability. It is assumed that thermal stability will be insured by the appropriate design of the dipole coils to provide for removal of the losses that are generated in the superconducting winding with an acceptably small rise in temperature during operation. Flux jump stability is related to conductor filament size and the electrical and thermal conductivity of the matrix through arguments of adiabatic and dynamic stabilization. Intrinsic, adiabatic or equivalently enthalpic stability is achieved in superconductors if the heat capacity of a local region of the conductor in the vicinity of a filament is sufficient to prevent the heat released during a flux jump from driving neighboring filaments in the superconductor normal. The maximum filament size which can be expected to be adiabatically stable is given roughly by the expression, (8)

$$
j_{c} d<\pi\left(\frac{\rho_{m} c_{p} T_{o}}{\mu_{0}}\right)^{1 / 2}
$$


where $c_{p}=$ the specific heat of the filament

$d=$ the diameter of the filament

$\rho_{m}=$ the density of the filament

$T_{0}=-j_{c} /\left(\partial j_{c} / \partial T\right)$.

The inclusion of high conductivity material in the matrix of the superconductor surrounding the filaments can result in an added, "dynamic" stabilization of the conductor. Eddy currents in the matrix damp the rate of entry of flux into the filaments during the flux $j$ ump, effectively decreasing the magnetic diffusivity relative to the thermal diffusivity in the composite and slowing the rate of heat generation relative to the time required for its removal.

The filaments in all of the superconductors being considered for the energy doubler are much smaller than the maximum allowed from the adiabatic stability formulae and dynamic stabilization with high conductivity matrix materials may not therefore be required. Experience has shown, however, that other factors which are not understood and depend upon the conductivity of the matrix material also influence conductor stability. Apparently, conductors are susceptible to disturbances of mechanical origin in the winding environment, ${ }^{(12)}$ and unexplained magnet quenches have often been classed as resulting from problems of mechanical instability.

The precise nature in which stability is affected mechanically is not known, but some comments can be made based upon a sense of the problem from information in the literature and discussion with individuals 
concerned with magnet design. The presence of a high conductivity matrix does seem to enhance the mechanical stability of conductors although the manner in which it acts is not clear. It may be that heat is produced by friction during conductor movement, or by the generation of heat as transient field losses due to movement of the conductor in a field gradient. In this case a high conductivity matrix may provide a shunting path during local partial normalization of the conductor which results from the transient thermal input. Improved stability should result from reduction of the joule heating which occurs in the partially normal conductor and improvement of heat transfer to the conductor surfaces and surroundings. A conductor design and heat treatment which maximize the axial conductivity and area of the stabilizing material, increases thermal conductivity within the conductor, and increases the surface area for heat transfer from the conductor seems desirable. The amount of resistive material introduced to lower losses, either through the construction of mixed matrix monolithic composites or solder impregnated cables should be minimized, and the conductivity of the stabilizing material should be maximized recognizing the limitations imposed by magnetoresistance and by the effect on the electronic mean free path of scattering from the filaments. (9) Caution should be exercised in the use of mixed matrix conductors since to the authors knowledge the stability of mixed matrix composites of the size and overall current density required for the energy doubler application has not been determined. 
$T M-545$

IV. Averaging Losses over Dipole Coils

The magnetic field distribution in the shell type dipole winding can be accurately determined by summing the fields produced by each shell. (10) Illustrated in Fig. I is a schematic of the shell type dipole winding. The magnetic fields produced by the $j$-th shell can be approximately calculated from

$$
\begin{aligned}
& r<a_{j i} \\
& H_{r}=-\sum_{n=\text { odd }} \frac{2 J_{j} \sin \left(\frac{n \phi_{i}}{2}\right) \sin (n \theta)}{n \pi(2-n)} r\left(\frac{r}{a_{j 0}}\right)^{n-2}\left(1-\left(\frac{a_{j i}}{a_{j 0}}\right)^{2-n}\right. \\
& \left.+\left(\frac{2-n}{2+n}\right)\left(\frac{a j o}{b}\right)^{2 n}\left[1-\left(\frac{a_{j i}}{a_{j o}}\right)^{2+n}\right]\right\} \\
& H_{\theta}=-\sum_{n=\text { odd }} \frac{2 J_{j} \sin \left(\frac{n \phi}{2}\right) \cos (n \theta)}{n \pi(2-n)} r\left(\frac{r}{a_{j 0}}\right)^{n-2}\left(1-\left(\frac{a_{j i}}{a_{j 0}}\right)^{2-n}\right. \\
& \left.+\left(\frac{2-n}{2+n}\right)\left(\frac{a_{j o}}{b}\right)^{2 n}\left[1-\left(\frac{a_{j i}}{a_{j 0}}\right)^{2+n}\right]\right\} \\
& a_{j i}<r<a_{j o} \\
& H_{r}=-\sum_{n=0 d d} \frac{2 J_{j} \sin \left(\frac{n \phi_{f}}{2}\right) \sin (n \theta)}{n \pi\left(4-n^{2}\right)} r\left\{-2 n-(2-n)\left(\frac{a_{j i}}{r}\right)^{n+2}\right. \\
& \left.+(2+n)\left(\frac{r}{a_{j 0}}\right)^{n-2}+(2-n)\left(\frac{r}{b}\right)^{n-2}\left(\frac{a_{j 0}}{b}\right)^{n+2}\left[1-\left(\frac{a_{j i}}{a_{j o}}\right)^{n+2}\right]\right\}
\end{aligned}
$$

9 


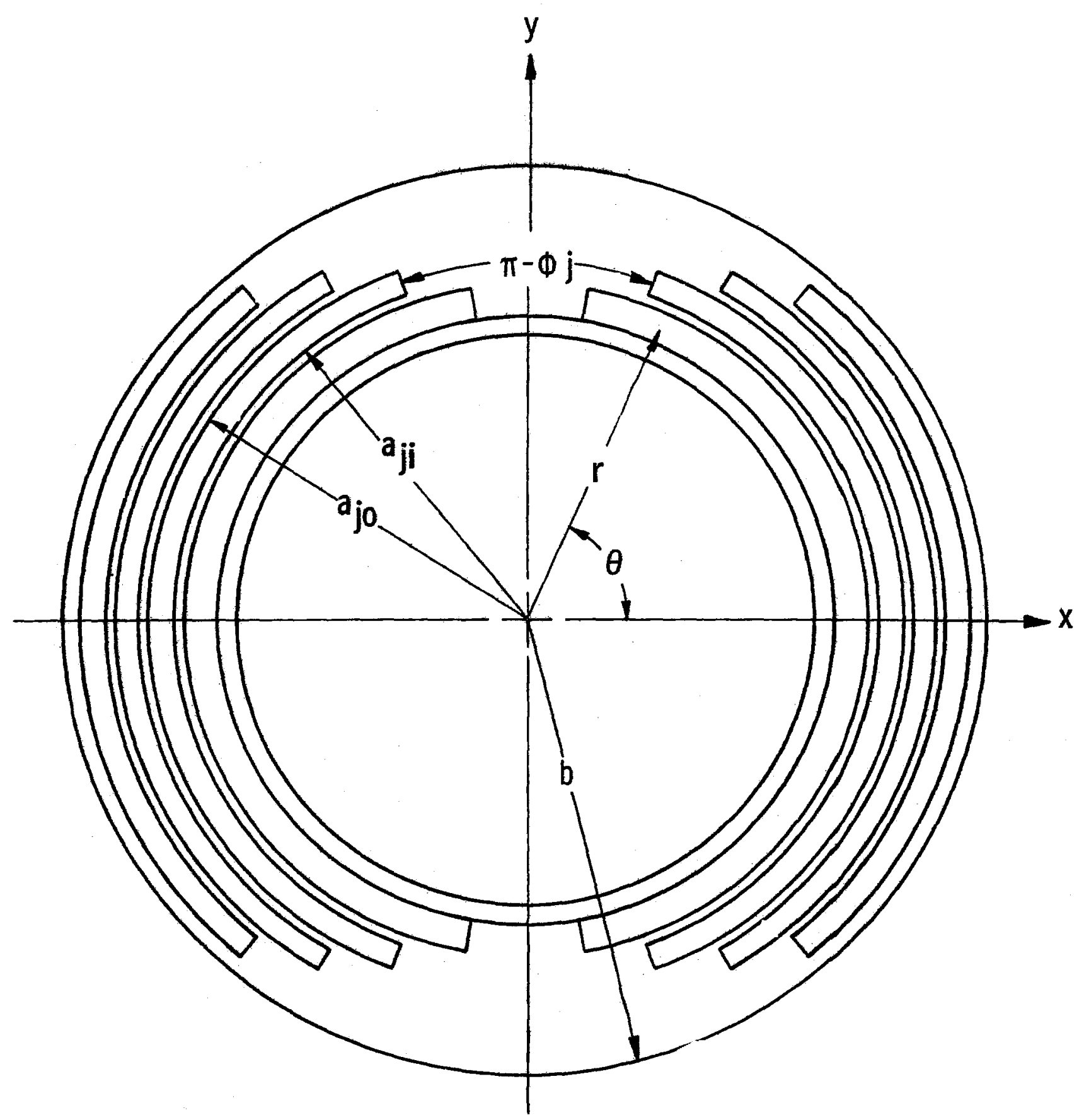

Fig. 1-Shell type dipole winding 


$$
\begin{aligned}
& H_{\theta}=-\sum_{n=0 d d} \frac{2 J_{j} \sin \left(\frac{n \phi}{2}\right) \cos (n \theta)}{n \pi\left(4-n^{2}\right)} r\left\{-4+(2-n p)\left(\frac{a_{j i}}{r}\right)^{n+2}\right. \\
& \left.+(2+n)\left(\frac{r}{a_{j 0}}\right)^{n-2}+(2-n)\left(\frac{r}{b}\right)^{n-2}\left(\frac{a_{j 0}}{b}\right)^{n+2}\left[1-\left(\frac{a_{j i}}{a_{j 0}}\right)^{n+2}\right]\right\}
\end{aligned}
$$

$a_{j 0}<r<b$

$$
\begin{aligned}
& H_{R}=-\sum_{n=o d d} \frac{2 J_{j} \sin \left(\frac{n \phi_{j}}{2}\right) \sin (n \theta)}{n_{\pi}(2+n)} \times\left(\frac{a_{j o}}{r}\right)^{n+2}\left[1-\left(\frac{a_{j i}}{a_{j o}}\right)^{n+2}\right]\left[+\left(\frac{r}{b}\right)^{2 n}\right] \\
& H_{\theta}=+\sum_{n=\text { odd }} \frac{2 J_{j} \sin \left(\frac{n \phi_{j}}{2}\right) \cos (n \theta)}{n \pi(2+n)} r\left(\frac{a_{j o}}{r}\right)^{n+2}\left[1-\left(\frac{a_{j i}}{a_{j o}}\right)^{n+2}\right]\left[1+\left(\frac{r}{b}\right)^{2 n}\right]
\end{aligned}
$$

where $H_{R}$ is the magnetic field in the radial direction at $r, \theta, H_{\theta}$ is the magnetic field in the circumferential direction at $r, \theta$, and $J_{j}$ is the average current density in the $j$-th shell. Equations (8) through (13) assumes that the iron ( $r \quad b)$ is not saturated and its relative permeability is large in comparison with unity.

Since the critical current density and the losses in the conductor are a function of the magnitude of the field on the wire, one must determine the field magnitude from

$$
H(r, \theta)=\left[\left(\sum_{j=1}^{m} H_{r}\right)^{2}+\left(\sum_{j=1}^{m} H_{\theta}\right)^{2}\right]^{1 / 2}
$$


where $m$ is the number of shells in the dipole winding.

In order to properly average the losses over the dipole windings one must determine the following three averaged quantities:

$$
\begin{aligned}
& \left\langle\left|H_{j}\right|\right\rangle=\frac{1}{\theta_{j}\left(a_{j o}{ }^{2}-a_{j i}{ }^{2}\right)} \\
& \int \\
& \text { Volume of } j \text {-th } \\
& \text { winding } \\
& \left\langle H_{j}^{2}\right\rangle=\frac{1}{\theta_{j}\left(a_{j 0}{ }^{2}-a_{j i}{ }^{2}\right)} \\
& \iint^{r} \\
& \text { Volume of } j \text {-th } \\
& \text { winding } \\
& \left\langle\frac{J_{j}}{J_{c}}\right\rangle=\frac{1}{\theta_{j}\left(a_{j 0}{ }^{2}-a_{j i}{ }^{2}\right)} \\
& \text { i } \\
& \frac{J_{j}}{J_{c}[H(r, \theta), T]} \\
& H^{2}(r, \theta) \operatorname{rdrd} \theta \\
& H(r, \theta) \operatorname{rdrd} \theta \\
& \text { Volume of } j \text {-th } \\
& \text { winding }
\end{aligned}
$$

for each shell in the dipole winding. The eddy current loss expression given by equation (3) should be averaged over the winding volume. If the major axis of the conductor is oriented radially then everywhere within the winding

$$
\tan \theta_{K}=\frac{H_{\theta}(r, \theta)}{H_{r}(r, \theta)}
$$

and the average quantities to be used in equation (3) are:

$$
\left\langle\sin ^{2}{ }_{\theta_{k}}\right\rangle=\frac{1}{\int_{j}\left(a_{j o}{ }^{2}-a_{j i}{ }^{2}\right)} \int_{\substack{\text { volume of } j-t h \\ \text { winding }}} \frac{H_{r}(r, \theta) r d r d \theta}{\sqrt{H_{r}^{2}(r, \theta)+H_{\theta}{ }^{2}(r, \theta)}}
$$


and

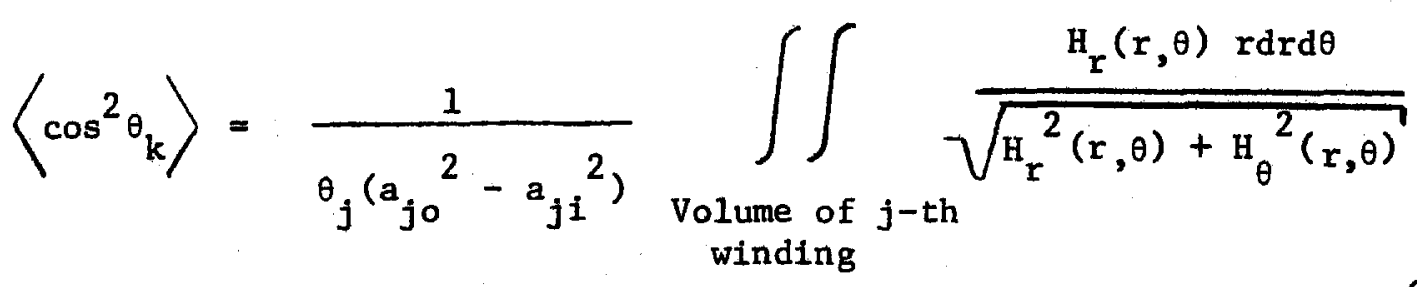




\section{Parametric Analysis}

There are many parameters which are important in the calculation of losses in a superconducting wire, as illustrated by equations (3) and (6). The relationship between these parameters can be easily determined from nomographs if one fixes some of the parameters.

The critical current density which can be carried by the superconductor depends upon the composition and heat treatment of the superconductor. Illustrated in Fig. 2 is a normalized graph of $j_{c}$ versus $H$ for typical NbTi superconductors. This graph allows one to estimate the average value of $j_{c}$ which must be incorporated in the hysteresis loss equations.

The eddy current loss in a rectangular conductor depends upon the twist pitch of the filaments, direction of the magnetic field, and the aspect ratio of the rectangle. The eddy current losses were calculated from equation (3). Table 1 ists the eddy current losses in cylindrical conductors for the several ramp rates which may be anticipated in the energy doubler and the peak field losses in rectangular conductors have been plotted in nomographs including the effect of rectangling as shown in Figs. 3-5. (A straight line through any two parameters gives the third parameter.)

For the ramped fields of interest to the energy doubler, the major hysteresis loss is a filamentary hysteresis loss. The hysteresis losses in the analysis which follows were calculated from equation (6) 


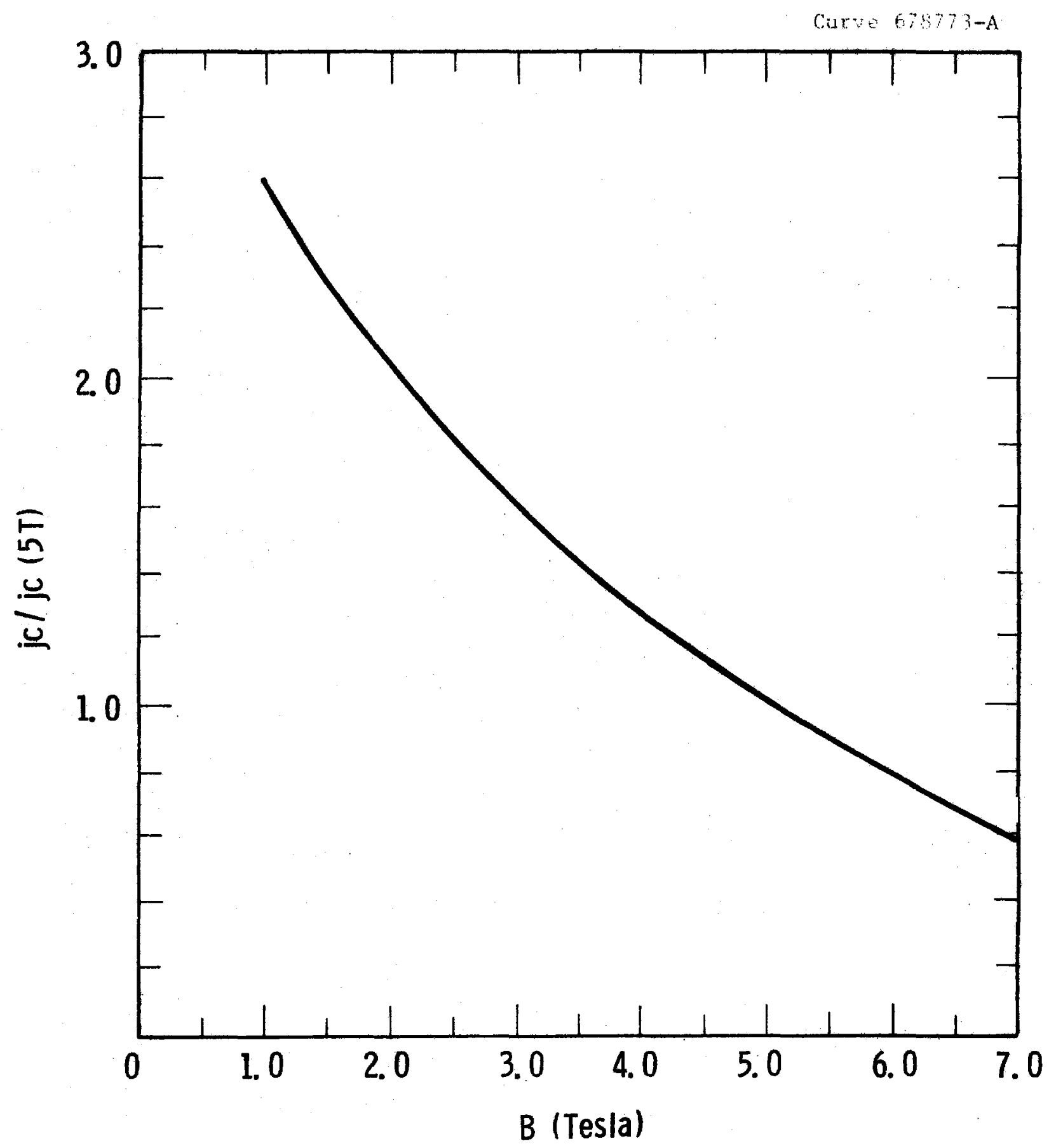

Fig. 2-Normalized $\mathrm{J}_{\mathrm{C}}-\mathrm{H}$ curve for NbTi superconductors 
Table 1 Eddy Current Losses in Superconducting Composites (watts/cubic centimeter)

(a) $B_{0}=6.25 \times 10^{3}$ Gauss $/ \sec \left(\frac{a}{b}\right)=1$

\begin{tabular}{|c|c|c|c|c|c|c|c|}
\hline $\begin{array}{c}\text { Composite } \\
\text { Resistivity } \\
(\mu \Omega \mathrm{cm})\end{array}$ & 0.2 & 0.5 & 1 & 2 & 5 & 10 & 20 \\
\hline 0.05 & $7.94 \times 10^{-5}$ & $4.95 \times 10^{-4}$ & $1.98 \times 10^{-3}$ & $7.94 \times 10^{-3}$ & $4.95 \times 10^{-2}$ & $1.98 \times 10^{-1}$ & $7.94 \times 10^{-1}$ \\
\hline 0.10 & $3.96 \times 10^{-5}$ & $2.48 \times 10^{-4}$ & $9.88 \times 10^{-4}$ & $3.96 \times 10^{-3}$ & $2.48 \times 10^{-2}$ & $9.88 \times 10^{-2}$ & $3.96 \times 10^{-1}$ \\
\hline 0.5 & $7.94 \times 10^{-6}$ & $4.95 \times 10^{-5}$ & $1.98 \times 10^{-4}$ & $7.94 \times 10^{-4}$ & $4.95 \times 10^{-3}$ & $1.98 \times 10^{-2}$ & $7.94 \times 10^{-2}$ \\
\hline 1.0 & $3.96 \times 10^{-6}$ & $2.48 \times 10^{-5}$ & $9.88 \times 10^{-5}$ & $3.96 \times 10^{-4}$ & $2.48 \times 10^{-3}$ & $9.88 \times 10^{-3}$ & $3.96 \times 10^{-2}$ \\
\hline 5.0 & $7.94 \times 10^{-7}$ & $4.95 \times 10^{-6}$ & $1.98 \times 10^{-5}$ & $7.94 \times 10^{-5}$ & $4.95 \times 10^{-4}$ & $1.98 \times 10^{-3}$ & $7.94 \times 10^{-3}$ \\
\hline 10.0 & $3.96 \times 10^{-7}$ & $2.48 \times 10^{-6}$ & $9.88 \times 10^{-6}$ & $3.96 \times 10^{-5}$ & $2.48 \times 10^{-4}$ & $9.88 \times 10^{-4}$ & $3.96 \times 10^{-3}$ \\
\hline
\end{tabular}

(b) $B_{0}=2.5 \times 10^{3}$ gauss $/ \mathrm{sec},\left(\frac{a}{b}\right)=1$

\begin{tabular}{|c|c|c|c|c|c|c|c|}
\hline $\begin{array}{c}\text { Composite } \\
\text { Resistivity } \\
(\mu \Omega \mathrm{cm})\end{array}$ & 0.2 & 0.5 & 1 & 2 & 5 & 10 & 20 \\
\hline 0.05 & $1.27 \times 10^{-5}$ & $7.92 \times 10^{-5}$ & $3.17 \times 10^{-4}$ & $1.27 \times 10^{-3}$ & $7.92 \times 10^{-3}$ & $3.17 \times 10^{-2}$ & $1.27 \times 10^{-1}$ \\
\hline 0.10 & $6.33 \times 10^{-6}$ & $3.96 \times 10^{-5}$ & $1.58 \times 10^{-4}$ & $6.33 \times 10^{-4}$ & $3.96 \times 10^{-3}$ & $1.58 \times 10^{-2}$ & $6.33 \times 10^{-2}$ \\
\hline 0.5 & $1.27 \times 10^{-6}$ & $7.92 \times 10^{-6}$ & $3.17 \times 10^{-5}$ & $1.27 \times 10^{-4}$ & $7.92 \times 10^{-4}$ & $3.17 \times 10^{-3}$ & $1.27 \times 10^{-2}$ \\
\hline 1.0 & $6.33 \times 10^{-7}$ & $3.96 \times 10^{-6}$ & $1.58 \times 10^{-5}$ & $6.33 \times 10^{-5}$ & $3.96 \times 10^{-4}$ & $1.58 \times 10^{-3}$ & $6.33 \times 10^{-3}$ \\
\hline 5.0 & $1.27 \times 10^{-7}$ & $7.92 \times 10^{-7}$ & $3.17 \times 10^{-6}$ & $1.27 \times 10^{-5}$ & $7.92 \times 10^{-5}$ & $3.17 \times 10^{-4}$ & $1.27 \times 10^{-3}$ \\
\hline 10.0 & $6.33 \times 10^{-8}$ & $3.96 \times 10^{-7}$ & $1.58 \times 10^{-6}$ & $6.33 \times 10^{-6}$ & $3.96 \times 10^{-5}$ & $1.58 \times 10^{-4}$ & $6.33 \times 10^{-4}$ \\
\hline
\end{tabular}

(c) $B_{0}=8.33 \times 10^{2}$ gauss $/$ sec, $\left(\frac{a}{b}\right)=1$

\begin{tabular}{|c|c|c|c|c|c|c|c|}
\hline $\begin{array}{c}\text { Composite } \\
\text { Resistivity } \\
(\mu \Omega \mathrm{cm})\end{array}$ & 0.2 & 0.5 & 1 & 2 & 5 & 10 & 20 \\
\hline 0.05 & $1.41 \times 10^{-6}$ & $8.80 \times 10^{-6}$ & $3.52 \times 10^{-5}$ & $1.41 \times 10^{-4}$ & $8.80 \times 10^{-4}$ & $3.52 \times 10^{-3}$ & $1.41 \times 10^{-2}$ \\
\hline 0.10 & $7.03 \times 10^{-7}$ & $4.40 \times 10^{-6}$ & $1.76 \times 10^{-5}$ & $7.03 \times 10^{-5}$ & $4.40 \times 10^{-4}$ & $1.76 \times 10^{-3}$ & $7.03 \times 10^{-3}$ \\
\hline 0.5 & $1.41 \times 10^{-7}$ & $8.80 \times 10^{-7}$ & $3.52 \times 10^{-6}$ & $1.41 \times 10^{-5}$ & $8.80 \times 10^{-5}$ & $3.52 \times 10^{-4}$ & $1.41 \times 10^{-3}$ \\
\hline 1.0 & $7.03 \times 10^{-8}$ & $4.40 \times 10^{-7}$ & $1.76 \times 10^{-6}$ & $7.03 \times 10^{-6}$ & $4.40 \times 10^{-5}$ & $1.76 \times 10^{-4}$ & $7.03 \times 10^{-4}$ \\
\hline 5.0 & $1.41 \times 10^{-8}$ & $8.80 \times 10^{-8}$ & $3.52 \times 10^{-7}$ & $1.41 \times 10^{-6}$ & $8.80 \times 10^{-6}$ & $3.52 \times 10^{-5}$ & $1.41 \times 10^{-4}$ \\
\hline 10.0 & $7.03 \times 10^{-9}$ & $4.40 \times 10^{-8}$ & $1.76 \times 10^{-7}$ & $7.03 \times 10^{-7}$ & $4.40 \times 10^{-6}$ & $1.76 \times 10^{-5}$ & $7.03 \times 10^{-5}$ \\
\hline
\end{tabular}




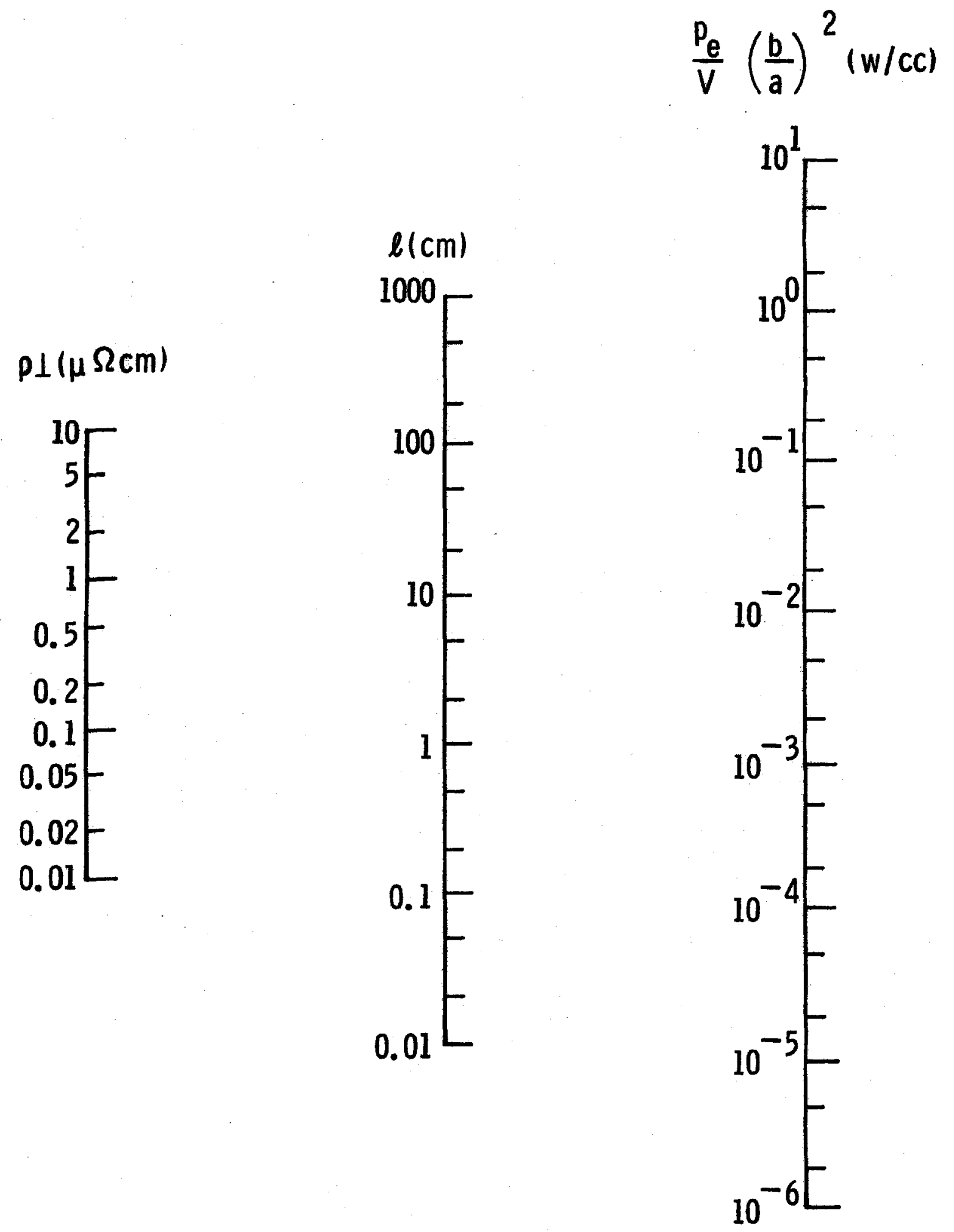

Fig. 3-Maximum Eddy current losses in superconducting composites for $\dot{B}_{0}=6.25 \times 10^{3}$ gauss $/ \mathrm{sec}$. 

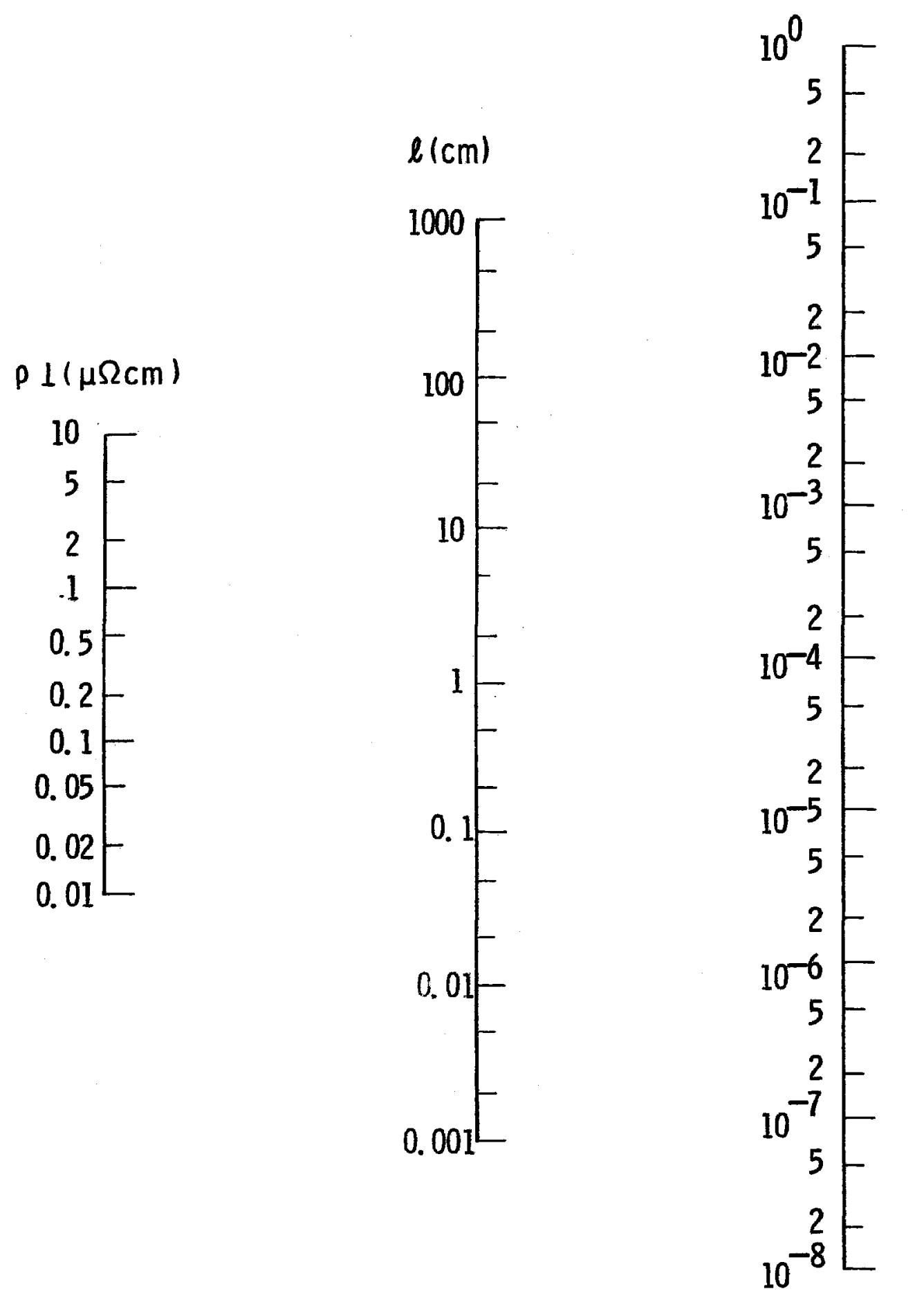

$$
\frac{P e}{V}\left(\frac{b}{a}\right)^{2} \quad(w / c c)
$$

Fig. 4-Maximum eddy current losses in superconducting composites for $\dot{\mathrm{B}}_{0}=2.5 \times 10^{3}$ gauss $/ \mathrm{sec}$ 

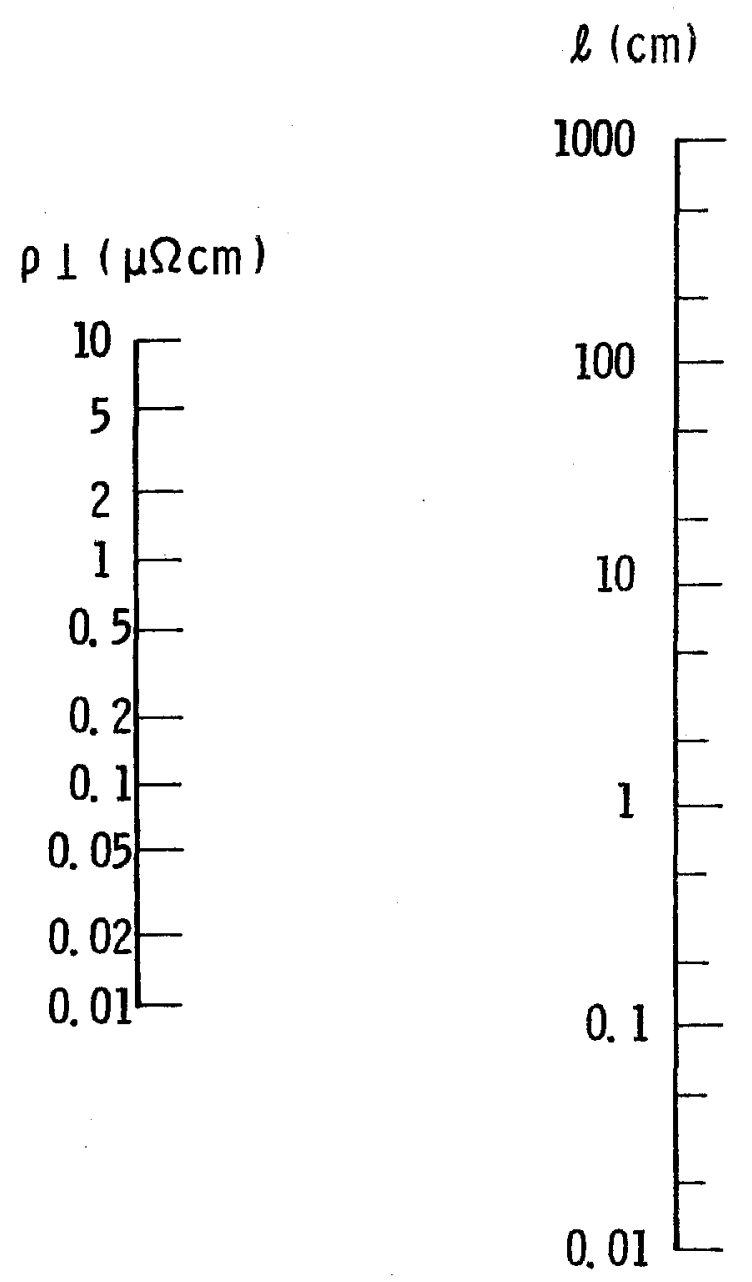

$$
\frac{P_{e}}{V}\left(\frac{b}{a}\right)^{2} \quad(w / c c)
$$

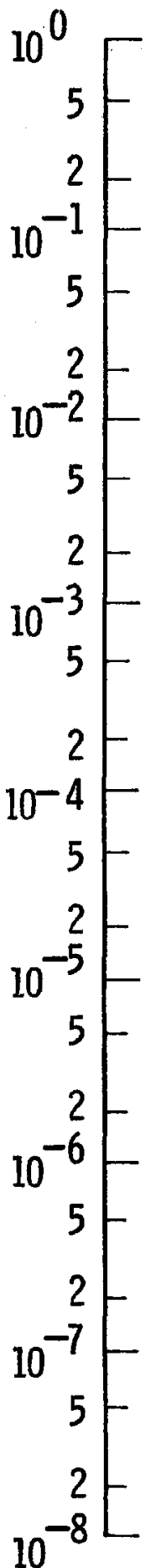

Fig. 5-Maximum eddy current losses in superconducting composites for $\dot{B}_{0}=8.33 \times 10^{2}$ gauss $/ \mathrm{sec}$ 
which presumes that a filament is carrying a transport current. This gives the maximum loss which can be anticipated and may be reduced by at most a factor of $3 \pi / 4$ depending upon the distribution of current in the conductor. Table 2 lists the filamentary hysteresis losses for the several pulse periods and these have been plotted in nomographs shown in Figs. 6-8. 
Table 2 Filamentary Hysteresis Losses at Maximum Transport Current (watts/cubic centimeter)

(a) $B_{0}=6.25 \times 10^{3}$ gauss $/ \mathrm{sec}$

\begin{tabular}{|c|c|c|c|c|c|c|c|}
\hline $\begin{array}{c}\text { Filament } \\
\text { Size } \\
(\mu \mathrm{m})\end{array}$ & $10^{4}$ & $2 \times 10^{4}$ & $5 \times 10^{4}$ & $10^{5}$ & $2 \times 10^{5}$ & $5 \times 10^{5}$ & $10^{6}$ \\
\hline 5 & $1.56 \times 10^{-4}$ & $3.08 \times 10^{-4}$ & $7.80 \times 10^{-4}$ & $1.56 \times 10^{-3}$ & $3.08 \times 10^{-3}$ & $7.80 \times 10^{-3}$ & $1.56 \times 10^{-2}$ \\
\hline 10 & $3.08 \times 10^{-4}$ & $6.16 \times 10^{-4}$ & $1.54 \times 10^{-3}$ & $3.08 \times 10^{-3}$ & $6.16 \times 10^{-3}$ & $1.54 \times 10^{-2}$ & $3.08 \times 10^{-2}$ \\
\hline 20 & $6.15 \times 10^{-4}$ & $1.23 \times 10^{-3}$ & $3.08 \times 10^{-3}$ & $6.15 \times 10^{-3}$ & $1.23 \times 10^{-2}$ & $3.08 \times 10^{-2}$ & $6.15 \times 10^{-2}$ \\
\hline 50 & $1.56 \times 10^{-3}$ & $3.08 \times 10^{-3}$ & $7.8 \times 10^{-3}$ & $1.56 \times 10^{-2}$ & $3.08 \times 10^{-2}$ & $7.80 \times 10^{-2}$ & $1.56 \times 10^{-1}$ \\
\hline
\end{tabular}

(b) $B_{0}=2.5 \times 10^{3}$ guass $/ \mathrm{sec}$

\begin{tabular}{|c|c|c|c|c|c|c|c|}
\hline $\begin{array}{c}\text { Filament } \\
\text { Size } \\
(\mu \mathrm{m})\end{array}$ & $10^{4}$ & $2 \times 10^{4}$ & $5 \times 10^{4}$ & $10^{5}$ & $2 \times 10^{5}$ & $5 \times 10^{5}$ & $10^{6}$ \\
\hline 5 & $6.25 \times 10^{-5}$ & $1.23 \times 10^{-4}$ & $3.13 \times 10^{-4}$ & $6.25 \times 10^{-4}$ & $1.23 \times 10^{-3}$ & $3.13 \times 10^{-3}$ & $6.25 \times 10^{-3}$ \\
\hline 10 & $1.23 \times 10^{-4}$ & $2.46 \times 10^{-4}$ & $6.25 \times 10^{-4}$ & $1.23 \times 10^{-3}$ & $2.46 \times 10^{-3}$ & $6.25 \times 10^{-3}$ & $1.23 \times 10^{-2}$ \\
\hline 20 & $2.46 \times 10^{-4}$ & $4.92 \times 10^{-4}$ & $1.23 \times 10^{-3}$ & $2.46 \times 10^{-3}$ & $4.92 \times 10^{-3}$ & $1.23 \times 10^{-2}$ & $2.46 \times 10^{-2}$ \\
\hline 50 & $6.25 \times 10^{-4}$ & $1.23 \times 10^{-3}$ & $3.13 \times 10^{-3}$ & $6.25 \times 10^{-3}$ & $1.23 \times 10^{-2}$ & $3.13 \times 10^{-2}$ & $6.25 \times 10^{-2}$ \\
\hline
\end{tabular}

(c) $B_{0}=8.33 \times 10^{2}$ gauss $/ \mathrm{sec}$

\begin{tabular}{|c|c|c|c|c|c|c|c|}
\hline $\begin{array}{c}\text { Filament } \\
\text { Size } \\
(\mu \mathrm{m})\end{array}$ & $10^{4}$ & $2 \times 10^{4}$ & $5 \times 10^{4}$ & $10^{5}$ & $2 \times 10^{5}$ & $5 \times 10^{5}$ & $10^{6}$ \\
\hline 5 & $2.08 \times 10^{-5}$ & $4.17 \times 10^{-5}$ & $1.04 \times 10^{-4}$ & $2.08 \times 10^{-4}$ & $4.17 \times 10^{-4}$ & $1.04 \times 10^{-8}$ & $2.08 \times 10^{-3}$ \\
\hline 5 & $4.17 \times 10^{-5}$ & $8.33 \times 10^{-5}$ & $2.08 \times 10^{-4}$ & $4.17 \times 10^{-4}$ & $8.33 \times 10^{-4}$ & $2.08 \times 10^{-3}$ & $4.17 \times 10^{-3}$ \\
\hline 10 & $8.33 \times 10^{-5}$ & $1.67 \times 10^{-4}$ & $4.17 \times 10^{-4}$ & $8.33 \times 10^{-4}$ & $1.67 \times 10^{-3}$ & $4.17 \times 10^{-3}$ & $8.33 \times 10^{-3}$ \\
\hline 20 & $2.08 \times 10^{-4}$ & $4.17 \times 10^{-4}$ & $1.04 \times 10^{-3}$ & $2.08 \times 10^{-3}$ & $4.17 \times 10^{-3}$ & $1.04 \times 10^{-2}$ & $2.08 \times 10^{-2}$ \\
\hline 50 & & &
\end{tabular}


Dwg. $6252 A 24$

$\overline{\lambda j}_{c}\left(a / \mathrm{cm}^{2}\right)$

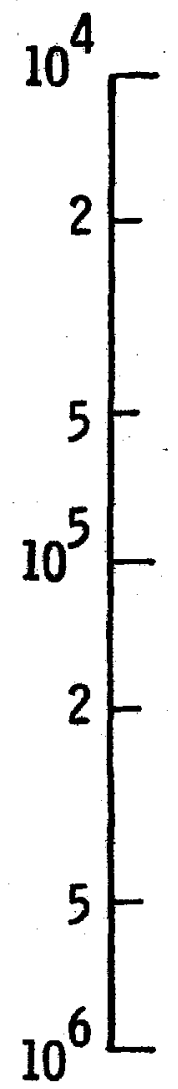

$P_{h} / V(w / c c)$

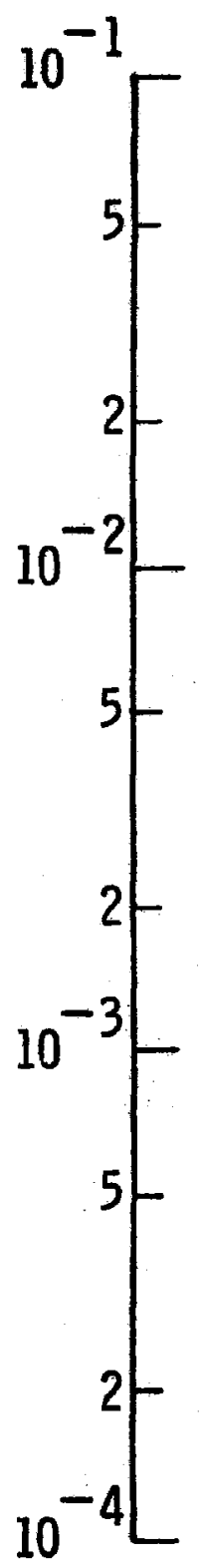

Fig. 6-Filamentary hysteresis loss at maximum transport current for $\dot{B}_{0}=6.25 \times 10^{3}$ gauss $/ \mathrm{sec}$ 

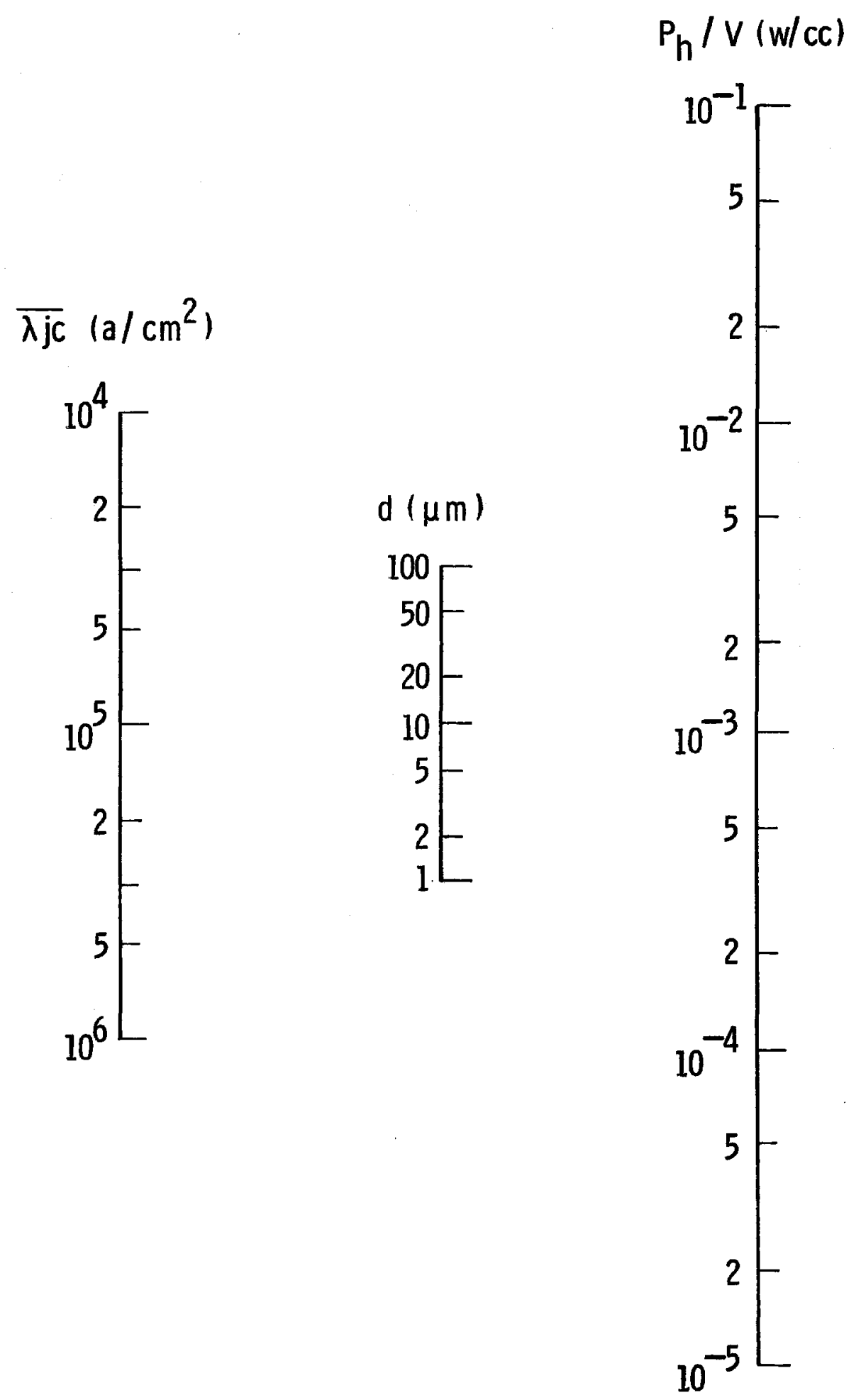

Fig. 7-Filamentary hysteresis loss at maximum transport current for $B_{0}=2.5 \times 10^{3}$ gauss $/ \mathrm{sec}$ 

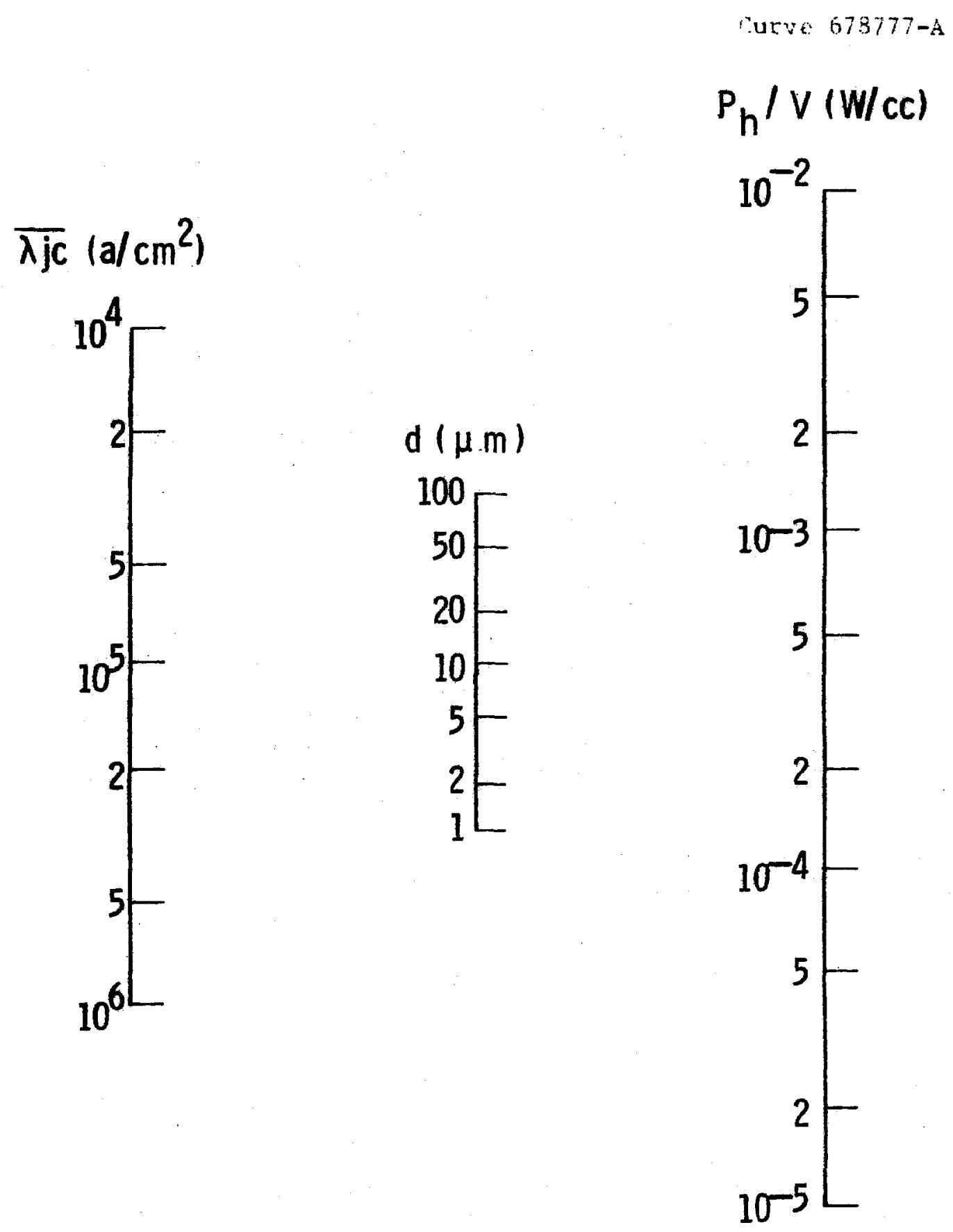

Fig. 8-Filamentary hysteresis loss at maximum transport current for $\dot{B}_{0}=833 \times 10^{2}$ gauss $/ \mathrm{sec}$ 


\section{Loss Calculations for Example Conductors}

The detailed geometry of the superconducting dipole is normally used to determine the loss per unit length as described in Section IV. However, for the purpose of this report only the peak field loss (i.e., based upon the bore field) is determined, and in order to estimate the total loss per unit length it will be assumed that a $20 \mathrm{ft}$ coil consists of $6.5 \times 10^{3} \mathrm{~cm}^{3}$ of the $0.075^{\prime \prime} \times 0.150^{\prime \prime}$ conductor and $6.5 \times 10^{3} \mathrm{~cm}^{3}$ of the $0.050 " \mathrm{x} 0.150^{\prime \prime}$ conductor. These conductors are designed to have a critical current of 3500 amps at 5 Tesla and have an operating current of 2300 amps at 4.5 Tesla. The critical current density which can be carried by the superconductor depends upon the composition and heat treatment of the conductor. For the conductors being considered for the energy doubler program a high-j $j_{c}$ superconductor $\left(j_{c}\right.$ at 5 tesla and $4.2^{\circ} \mathrm{K}$ of $1.84 \times 10^{5} \mathrm{a} / \mathrm{cm}^{2}$ ) has been proposed. In the following calculations it has been assumed that this type of material will be used.

The adiabatic stability criterion discussed in Section III [equation (7)] requires that the filament size be less than $96 \mu \mathrm{m}$ for the conductor to be stable against a flux jump at 2 Tesla. In this calculation for a conductor operating at $4.2^{\circ} \mathrm{K}$, it was assumed that $\rho_{\mathrm{m}} \mathrm{C}_{\mathrm{p}}=4 \times 10^{3} \mathrm{j} / \mathrm{m}^{3}-{ }^{\circ} \mathrm{K}$ and that $\mathrm{T}_{\mathrm{o}} \sim 4.0^{\circ} \mathrm{K} .{ }^{(8)}$ A11 of the conductors considered have filaments small compared to this value.

Table 3 lists the conductors analyzed in this report and describes their geometries. 
Table 3 Conductor Descriptions

\begin{tabular}{|c|c|c|c|c|c|c|c|c|c|c|c|}
\hline No. & Type & Size. & $\begin{array}{l}\text { Composite } \\
\text { Twist Length }\end{array}$ & $\begin{array}{l}\text { No. } \\
\text { Strands }\end{array}$ & $\begin{array}{l}\text { Filaments } \\
\text { per Strand }\end{array}$ & $\begin{array}{l}\text { Strand } \\
\text { Twist Length }\end{array}$ & $\begin{array}{l}\text { Filament } \\
\text { Size (um) }\end{array}$ & $\frac{p_{1}{ }^{0}}{\left(u \Omega_{\mathrm{cm}}\right)}$ & $\frac{0_{1} 1}{(\mu \sqrt{2} \mathrm{~cm})}$ & $\begin{array}{l}\text { Composite } \\
\text { Composition }\end{array}$ & $\begin{array}{l}\text { Strand } \\
\text { Composition }\end{array}$ \\
\hline 1 & Monolithic & $\begin{array}{r}0.075^{\prime \prime} \\
\times 0.150^{\prime \prime} \\
\end{array}$ & $0.75^{\prime \prime}$ & 1 & 3000 & -- & 28.4 & $2.8 \times 10^{-2}$ & - & Copper Matrix & $\because$ \\
\hline 2 & Monolithic & $\begin{array}{r}0.050^{\prime \prime} \\
\times 0.150^{\prime \prime} \\
\end{array}$ & $0.50^{\prime \prime}$ & 1 & 3000 & - & 23.2 & $2.8 \times 10^{-2}$ & - & Copper Matrix & -- \\
\hline 3 & Monolithic & $\begin{array}{r}0.075^{\prime \prime} \\
\times 0.150^{\prime \prime} \\
\end{array}$ & $0.75^{\prime \prime}$ & 1 & 3000 & - & 28.4 & 5.0 & - & $\begin{array}{c}\text { Cu30\%N1: Cu: Mb Ti } \\
1: 2: 1\end{array}$ & - \\
\hline 4 & Monoll thic & $\begin{array}{r}0.050^{\prime \prime} \\
\times 0.150^{\prime \prime} \\
\end{array}$ & $0.50^{\prime \prime}$ & 1 & 3000 & -- & 23.2 & 5.0 & -- & $\begin{array}{l}\text { Cu30\%Ni: Cu: NbTi } \\
1: 2: 1\end{array}$ & -- \\
\hline 5 & Monolithic & $\begin{array}{r}0.075^{\prime \prime} \\
\times 0.150^{\prime \prime} \\
\end{array}$ & $0.75^{\prime \prime}$ & 1 & 3000 & $\cdots$ & 28.4 & $5.1 \times 10^{-2}$ & - & $\begin{array}{c}\text { Cu:Cu30\%Ni:NbTi } \\
2: 1: 1 \\
\end{array}$ & -- \\
\hline 6 & Monolithic & $\begin{array}{r}0.050^{\prime \prime} \\
\times 0.150^{\prime \prime} \\
\end{array}$ & $0.50^{\prime \prime}$ & $\mathrm{I}$ & 3000 & -- & 23.2 & $5.1 \times 10^{-2}$ & -- & $\begin{array}{c}\text { Cu: Cu30\%N1:NbT1 } \\
2: 1: 1 \\
\end{array}$ & - \\
\hline 7 & Cable & $\begin{array}{r}0.075^{\prime \prime} \\
\times 0.150^{\prime \prime} \\
\end{array}$ & $5 \mathrm{~cm}$ & 8 & 2100 & $1 \mathrm{~cm}$ & 12.0 & 4.21 & $3.4 \times 10^{-2}$ & Solder Filled & $\begin{array}{l}\text { Cu Matrix, } \\
\text { CuN1 Sheach }\end{array}$ \\
\hline 8 & Cable & $\begin{array}{r}0.050^{\prime \prime} \\
\times 0.150^{\prime \prime} \\
\end{array}$ & $5 \mathrm{~cm}$ & 12 & 2100 & $0.5 \mathrm{~cm}$ & 8.0 & 4,21 & $3.4 \times 10^{-2}$ & Solder Filled & $\begin{array}{l}\text { Cu Matrix, } \\
\text { CuNi Sheath }\end{array}$ \\
\hline 9 & Cable & $\begin{array}{r}0.075^{\prime \prime} \\
\times 0.150^{\prime \prime} \\
\end{array}$ & $0.75^{\prime \prime}$ & 7 & 2400 & $0.375^{\prime \prime}$ & 12.0 & 6.47 & 5.0 & Solder Filled & $\begin{array}{c}\text { Cu30\%Ni: Cu:NbTi } \\
2: 3: 3\end{array}$ \\
\hline 10 & Cable & $\begin{array}{r}0.050^{\prime \prime} \\
\times 0.150^{\prime \prime} \\
\end{array}$ & $0.50^{\prime \prime}$ & 11 & 2400 & $0.25^{\prime \prime}$ & 8.0 & 5.70 & 5.0 & Solder Filled & $\begin{array}{l}\text { Cu } 30 \% \mathrm{Ni}: \mathrm{Cu}: \mathrm{Nb} \mathrm{Ti} \\
2: 3: 3\end{array}$ \\
\hline 11 & Cable & $\begin{array}{r}0.075^{\prime \prime} \\
\times 0.150^{\prime \prime} \\
\end{array}$ & $0.75^{\prime \prime}$ & 7 & 2400 & $0.375^{\prime \prime}$ & 12.0 & 6.47 & $3.79 \times 10^{-2}$ & Solder Filled & $\begin{array}{l}\text { Cu Macrix, } \\
\text { CuNi Sheath }\end{array}$ \\
\hline 12 & Cable & $\begin{array}{r}0.050^{\prime \prime} \\
\times 0.150^{\prime \prime} \\
\end{array}$ & $0.50^{\prime \prime}$ & 11 & 2400 & $0.25^{\prime \prime}$ & 8.0 & 5.70 & $3.79 \times 10^{-2}$ & Solder filled & $\begin{array}{l}\text { Cu Matrix, } \\
\text { CuN1 Sheath } \\
\end{array}$ \\
\hline 13 & Monolithic & $\begin{array}{r}0.075^{\prime \prime} \\
\times 0.150^{\prime \prime} \\
\end{array}$ & $0.75^{\prime \prime}$ & 8. & 375 & $0.75^{\prime \prime}$ & 28.4 & 5.0 & $3.4 \times 10^{-2}$ & $\begin{array}{r}\text { 8Cu30ZN1 Barrlers } \\
\text { Cu30\%N1:NbTi 1:1 }\end{array}$ & Copper Matrix \\
\hline 14 & Monolithic & $\begin{array}{r}0.050^{\prime \prime} \\
\times 0.150^{\prime \prime} \\
\end{array}$ & $0.50^{\prime \prime}$ & 8 & 375 & $0.50^{\prime \prime}$ & 23.2 & 5.0 & $3.4 \times 10^{-2}$ & $\begin{array}{r}\text { 8Cu30\%N1 Barriers } \\
\text { Cu 30\%Ni:NbTi } 1: 1\end{array}$ & Coppet Matrix \\
\hline
\end{tabular}


Illustrated in Tables 4 through 6 are conductor loss calculations for 30,10 and 4 second ramps. For these tables, it was assumed that $\rho_{\mathrm{m}}$ for copper to $1.7 \times 10^{-2} \mu \Omega \mathrm{cm}$, and for the resistive solder and Cu30\% $\mathrm{Ni} \rho_{m}$ is $35 \mu \Omega \mathrm{cm}$. The eddy current losses in the cabled conductors have been broken down into two components. The first component listed, $\mathrm{P}_{\text {eo }} / \mathrm{V}$ is the intrastrand eddy current loss and is calculated from equation (3) using the cable twist length. The second component listed, $\mathrm{P}_{\mathrm{el}} / \mathrm{V}$ is the interstrand eddy current loss calculated from equations (2) and (3) using the strand twist length and averaging over the entire conductor volume.

Equation (1) was used for determination of the transverse conductivity for copper matrices and equation (2) was used for cases of copper nicke1 matrices.

Although $\rho_{\perp_{0}}$ was calculated using equation (1) assuming a high resistivity solder for the cables, the same values for $\rho_{\perp_{0}}$ can be obtained with higher conductivity solders providing a resistive sheath surrounds the strands. A rough guide to $\rho_{\perp_{0}}$ is given by the formula $\rho_{\perp 0} \sim \frac{t}{R_{0}} \rho_{s}$ where $t$ is the thickness of the sheath around the strands, $P_{S}$ is the sheath resistivity and $R_{0}$ is the strand radius.

Since the continuum model describes eddy current losses between large numbers of superconducting elements in a normally conducting medium, it will not accurately describe strand to strand eddy currents for the few strands used in the cables. The formula should nevertheless provide a reasonable rough estimate of losses for the cable situation.

All conductors 1 isted meet the energy doubler requirements for a 30 second ramp. The metallic filled cable designs (conductors 
Table 4 Conductor Loss Calculations

30 Second Ramp, $B_{0}=8.33 \times 10^{2}$ gauss $/ \mathrm{sec}$

\begin{tabular}{|c|c|c|c|c|c|}
\hline No. & $\begin{array}{l}P_{e o} / V \\
(w / c c)\end{array}$ & $\begin{array}{c}\mathrm{P}_{\mathrm{el}} / \mathrm{V} \\
(\mathrm{w} / \mathrm{cc})\end{array}$ & $\begin{array}{r}P_{h} / V \\
(w / c c)\end{array}$ & $\begin{array}{c}P / V \\
(w / c c)\end{array}$ & $\begin{array}{c}P / l \\
(w / f t)\end{array}$ \\
\hline 1 & $9.16 \times 10^{-4}$ & -- & $8.47 \times 10^{-4}$ & $1.76 \times 10^{-3}$ & \multirow{2}{*}{1.10} \\
\hline 2 & $9.13 \times 10^{-4}$ & -- & $6.92 \times 10^{-4}$ & $1.61 \times 10^{-3}$ & \\
\hline 3 & $5.11 \times 10^{-6}$ & - & $8.47 \times 10^{-4}$ & $8.52 \times 10^{-4}$ & \multirow{2}{*}{0.503} \\
\hline 4 & $5.11 \times 10^{-6}$ & - & $6.92 \times 10^{-4}$ & $6.97 \times 10^{-4}$ & \\
\hline 5 & $5.02 \times 10^{-4}$ & - & $8.47 \times 10^{-4}$ & $1.35 \times 10^{-3}$ & \multirow{2}{*}{0.826} \\
\hline 6 & $5.01 \times 10^{-4}$ & $=$ & $6.92 \times 10^{-4}$ & $1.19 \times 10^{-3}$ & \\
\hline 7 & $4.18 \times 10^{-5}$ & $4.06 \times 10^{-5}$ & $3.74 \times 10^{-4}$ & $4.56 \times 10^{-4}$ & \multirow{2}{*}{0.263} \\
\hline 8 & $9.41 \times 10^{-5}$ & $1.01 \times 10^{-5}$ & $2.50 \times 10^{-4}$ & $3.54 \times 10^{-4}$ & \\
\hline 9 & $3.93 \times 10^{-6}$ & $2.20 \times 10^{-7}$ & $.3 .74 \times 10^{-4}$ & $3.78 \times 10^{-4}$ & \multirow{2}{*}{0.209} \\
\hline 10 & $4.47 \times 10^{-6}$ & $1.02 \times 10^{-7}$ & $2.61 \times 10^{-4}$ & $2.66 \times 10^{-4}$ & \\
\hline 11 & $3.93 \times 10^{-6}$ & $2.89 \times 10^{-5}$ & $3.74 \times 10^{-4}$ & $4.07 \times 10^{-4}$ & \multirow{2}{*}{0.223} \\
\hline 12 & $4.47 \times 10^{-6}$ & $1.35 \times 10^{-5}$ & $2.61 \times 10^{-4}$ & $2.79 \times 10^{-4}$ & \\
\hline 13 & $5.11 \times 10^{-6}$ & $1.40 \times 10^{-4}$ & $8.47 \times 10^{-4}$ & $9.92 \times 10^{-4}$ & \multirow{2}{*}{0.249} \\
\hline 14 & $5.11 \times 10^{-6}$ & $6.24 \times 10^{-5}$ & $6.92 \times 10^{-4}$ & $7.66 \times 10^{-4}$ & \\
\hline
\end{tabular}


Table 5 Conductor Loss Calculations 10 Second Ramp, $B_{0}=2.5 \times 10^{3}$ Gauss $/ \mathrm{Sec}$

\begin{tabular}{|c|c|c|c|c|c|}
\hline No. & $\begin{array}{l}\mathrm{P}_{\mathrm{eo}} / \mathrm{V} \\
(\mathrm{w} / \mathrm{cc})\end{array}$ & $\begin{array}{l}P_{e i} / V \\
(w / c c)\end{array}$ & $\begin{array}{c}\mathrm{P}_{\mathrm{h}} / \mathrm{V} \\
(\mathrm{w} / \mathrm{cc})\end{array}$ & $\begin{array}{c}P / V \\
(w / c c)\end{array}$ & $\begin{array}{l}P / \ell \\
(w / f t)\end{array}$ \\
\hline 1 & $8.24 \times 10^{-3}$ & - & $2.54 \times 10^{-3}$ & $1.08 \times 10^{-2}$ & \multirow{2}{*}{6.85} \\
\hline 2 & $8.22 \times 10^{-3}$ & $=$ & $2.07 \times 10^{-3}$ & $1.03 \times 10^{-2}$ & \\
\hline 3 & $4.60 \times 10^{-5}$ & - & $2.54 \times 10^{-3}$ & $2.59 \times 10^{-3}$ & \multirow{2}{*}{1.53} \\
\hline 4 & $4.60 \times 10^{-5}$ & -- & $2.07 \times 10^{-3}$ & $2.12 \times 10^{-3}$ & \\
\hline 5 & $4.52 \times 10^{-3}$ & $=$ & $2.54 \times 10^{-3}$ & $7.06 \times 10^{-3}$ & \multirow{2}{*}{4.43} \\
\hline 6 & $4.51 \times 10^{-3}$ & -- & $2.07 \times 10^{-3}$ & $6.58 \times 10^{-3}$ & \\
\hline 7 & $3.76 \times 10^{-4}$ & $3.65 \times 10^{-4}$ & $1.12 \times 10^{-3}$ & $1.86 \times 10^{-3}$ & \multirow{2}{*}{1.15} \\
\hline 8 & $8.47 \times 10^{-4}$ & $9.12 \times 10^{-5}$ & $7.50 \times 10^{-4}$ & $1.69 \times 10^{-3}$ & \\
\hline 9 & $3.54 \times 10^{-5}$ & $1.98 \times 10^{-6}$ & $1.12 \times 10^{-3}$ & $1.16 \times 10^{-3}$ & \multirow{2}{*}{0.644} \\
\hline 10 & $4.02 \times 10^{-5}$ & $9.22 \times 10^{-7}$ & $7.84 \times 10^{-4}$ & $8.25 \times 10^{-4}$ & \\
\hline 11 & $3.54 \times 10^{-5}$ & $2.60 \times 10^{-4}$ & $1.12 \times 10^{-3}$ & $1.42 \times 10^{-3}$ & \multirow{2}{*}{0.770} \\
\hline 12 & $4.02 \times 10^{-5}$ & $1.22 \times 10^{-4}$ & $7.84 \times 10^{-4}$ & $9.46 \times 10^{-4}$ & \\
\hline 13 & $4.60 \times 10^{-5}$ & $1.26 \times 10^{-3}$ & $2.54 \times 10^{-3}$ & $3.85 \times 10^{-3}$ & \multirow{2}{*}{2.12} \\
\hline 14 & $4.60 \times 10^{-5}$ & $5.62 \times 10^{-4}$ & $2.07 \times 10^{-3}$ & $2.68 \times 10^{-3}$ & \\
\hline
\end{tabular}


Table 6 Conductor Loss Calculations

4 Second Ramp, $B_{0}=6.25 \times 10^{3}$ Gauss $/$ Sec

\begin{tabular}{|c|c|c|c|c|c|}
\hline No. & $\begin{array}{l}P_{e o} / V \\
(w / c c)\end{array}$ & $\begin{array}{c}\mathrm{PP}_{\mathrm{ei}} / \mathrm{V} \\
(\mathrm{w} / \mathrm{cc})\end{array}$ & $\begin{array}{c}P_{h} / V \\
(w / c c)\end{array}$ & $\begin{array}{l}P / V \\
(w / c c)\end{array}$ & $\begin{array}{l}P / \ell \\
(w / f t)\end{array}$ \\
\hline 3 & $2.87 \times 10^{-4}$ & - & $6.4 \times 10^{-3}$ & $6.7 \times 10^{-3}$ & \multirow{2}{*}{3.94} \\
\hline 4 & $2.87 \times 10^{-4}$ & - & $5.23 \times 10^{-3}$ & $5.42 \times 10^{-3}$ & \\
\hline 7 & $2.28 \times 10^{-3}$ & $2.35 \times 10^{-3}$ & $2.8 \times 10^{-3}$ & $7.43 \times 10^{-3}$ & \multirow{2}{*}{4.93} \\
\hline 8 & $5.7 \times 10^{-4}$ & $5.29 \times 10^{-3}$ & $1.88 \times 10^{-3}$ & $7.74 \times 10^{-3}$ & \\
\hline 9 & $1.24 \times 10^{-5}$ & $2.21 \times 10^{-4}$ & $2.8 \times 10^{-3}$ & $3.03 \times 10^{-3}$ & \multirow{2}{*}{1.72} \\
\hline 10 & $5.49 \times 10^{-6}$ & $2.51 \times 10^{-4}$ & $1.96 \times 10^{-3}$ & $2.27 \times 10^{-3}$ & \\
\hline 11 & $1.63 \times 10^{-3}$ & $2.21 \times 10^{-4}$ & $2.8 \times 10^{-3}$ & $4.65 \times 10^{-3}$ & \multirow{2}{*}{2.48} \\
\hline 12 & $7.63 \times 10^{-4}$ & $2.51 \times 10^{-4}$ & $1.96 \times 10^{-3}$ & $2.97 \times 10^{-3}$ & \\
\hline
\end{tabular}


7 through 12) meet the energy doubler requirements for a 10 second ramp, and among the monolithic conductor designs, the mixed matrix conductors (conductors 3 and 4 ) with $\mathrm{Cu} 30 \% \mathrm{Ni}$ webbs surrounding copper clad filaments will probably meet the energy doubler requirements when pulsed in 10 seconds.

Losses for a few of the conductors which as basic types can be considered for the faster 4 second ramp time are presented in Table 6 . of these the cabled conductors 9 and 10 are close to meeting the doubler loss requirement and the dominant loss, which is hysteretic can be reduced by reducing filament size. Conductors 3 and 4 have sufficiently low eddy current losses so that reduction of hysteresis losses through the reduction of filament size will also permit operation within the loss requirements of the energy doubler program.

A comparison of the results obtained on conductors 3 through 6 and 13 and 14 illustrates where the Cu30\% Ni webbing is most effective. In conductors 13 and 14, groups of filaments in a copper matrix were separated by 8 "radia1" $\mathrm{Cu} 30 \% \mathrm{Ni}$ webbs. In conductors 5 and 6 , each filament is encased by a Cu30\% $\mathrm{Ni}$ sheath and distributed throughout the copper matrix. In conductors 3 and 4, each filament is copper clad and distributed throughout a Cu30\% $\mathrm{Ni}$ matrix. The most effective use of the Cu30\% $\mathrm{Ni}$ is achieved in conductors 3 and 4 where copper clad filaments are distributed throughout a $\mathrm{Cu} 30 \% \mathrm{Ni}$ matrix and the resistive matrix forms a webbing which is placed so that it can most effectively interrupt a current traveling between any two filaments in the array. 
It is important to emphasize at this point that both the eddy current and hysteresis losses quoted in this section represent upper bounds determined from equations (3) and (6). Many of the marginal conductor designs presented may in fact meet the energy doubler requirements because the actual loss is less than the loss based upon the bore field and the conductor is carrying a current which is less than the critical current.

The losses resulting from ripple fields in the coll should be very small compared to ramped field losses according to the following arguments. The largest possible ripple voltage that is anticipated for the dipole power supply is approximately $7 \%$ of the voltage available for ramping the coil, and $A$ in the coil will be nearly proportional to the applied voltage so that ( the hysteresis losses in equation (6) are proportional to $\mathrm{A}$ and for small ripple field changes hysteresis losses can be considerably reduced from equation (6), because of incomplete penetration of the filaments, (2) a ripple loss of less than $7 \%$ of the ramped field is expected. Eddy current losses are proportional to (H) ${ }^{2}$ according to equation (3) and should be even smaller if shielding or skin effects characteristic at higher frequencies are considered. (1) The eddy current loss due to ripple fields is accordingly negligible. 
VII. Conclusions and Recommendations

For the anticipated mode of operation of the energy doubler colls, ripple field losses should be small compared to those produced by the ramped field. The ramped field loss calculations which have been made are based upon round filaments and exposure of the conductor to the peak field in the coil normal to its wide side. Unless otherwise stated the conclusions and recommendations in this section are made conservatively assuming that this maximum loss will occur for conductor elements throughout the coil. However, those instances where the choice of type of conductor may be affected by a precise calculation of losses are pointed out. The guidelines and formulae provided in this report are sufficient to permit calculation using detailed information regarding conductor position, field strength and field direction. The conclusions and recommendations which have been derived from the present analysis are as follows:

(1) For the 30 second ramp the energy doubler loss requirement of less than one watt per foot of coil length can be achieved with a tightly twisted monolithic copper matrix conductor with about 3000 filaments (conductors 1 and 2). Although calculations have been carried out assuming high $\mathrm{J}_{\mathrm{C}}$ superconductors, it is recommended for stability that the use of high critical current density NbTi not be stressed if it is achieved at the risk of increased resistivity in the copper matrix. An increase in the fraction of superconductor can be easily incorporated in 
the design to accommodate a reduced $\mathrm{J}_{\mathrm{c}^{*}}$

(2) On the basis of both the hysteresis and eddy current losses which have been calculated the copper matrix conductor recommended above will not be acceptable for the ten second ramp. However, since this relatively unsophisticated copper matrix conductor is by far the cheapest and simplest of the possible conductor designs and the actual average coil loss may be substantially reduced from the peak loss reported here, it is recommended that a detailed loss study utilizing information on local fleld conditions throughout the coil and confirming measurements be carried out to determine its applicability for the shorter pulse time.

(3) If hysteresis losses are limiting in (2) they can be reduced in proportion to the decrease in filament size. This decrease can be effected without major increase in the conductor cost providing it can be accomplished without the restacking of multifilament rods in the manufacturing process.

(4) Reduction of the eddy current loss can be accomplished in two basic ways: (a) by introducing a resistive component which separates individual filaments, and (b) by reducing the twist pitch in resistivity isolated sections of the conductor. Thus, for example, losses are reduced in cabled conductors where strands are separated by resistive solder and the twist within each strand is less than the overall cable twist pitch, but losses are not reduced in monolithic conductors where groups of filaments are enclosed in a copper nickel webb because the twist pitch for each group is as long as that of the conductor.

(5) The investigation on determining the optimal location for resistive webbing has confirmed that the most effective use of the material 
is accomplished when copper clad filaments are distributed throughout a resistive matrix.

(6) If monolithic copper matrix conductors prove unacceptable for the ten second ramp, solder filled cables are recommended at the present stage of conductor development. Both hysteresis and eddy current losses can simultaneously be reduced through stranding to meet the energy doubler requirements. The particular cabled conductor which has been developed at NAL is recommended, but with smaller NbTi filaments in the strands depending upon the degree to which a reduction in hysteresis losses is desirable. The calculations in this report on this type of conductor (examples $\# 7$ and $\# 8$ ) indicate that twelve micron filaments will reduce the maximum losses to meet the average coil loss requirements, and that the eddy current loss is about a factor of four lower. The copper nickel sheath around each strand should be retained to insure against low resistance strand to strand coupling preferably the higher resistivity copper $-30 \%$ nickel alloy and it is recommended that attempts not be made to improve critical current in the NbTi beyond the equivalent of $\sim 10^{5} \mathrm{~A} / \mathrm{cm}^{2}$ at 50 kilogauss if there is risk of increasing resistance in the copper matrix. If increased margin in critical current is required, an increase in the fraction of superconductor is recommended. (7) The cable of copper matrix strands which have been recommended in (6) for the 10 second ramp time will exceed by about a factor of two the conductor loss requirement in both hysteresis and eddy current losses for the 4 second ramp on the basis of the maximum loss calculations presented here. Since, however, improvements over this cable design will involve the 
use of more sophistlcated conductors and factor of two differences between average and maximum losses in the coil are possible, it is recommended that a more refined calculation be made of the average loss taking into account the field strength and direction throughout the coil. If the losses are acceptable, then this cable is recommended for the four second as well as ten second ramp time, in view of the present state of the art in conductor development.

(8) If average loss calculations show that hysteresis losses are excessive for the cable of copper matrix strands, then strands containing a 1: rer number of smaller filaments is recommended for the four second ramp period.

(9) If eddy current losses are excessive, then a mixed matrix conductor consisting of a minimum amount of copper-10\% nickel surrounding individual copper stabilized filaments is recommended. Filaments of the order of ten microns in diameter are indicated. Although there has been some difficulty in providing filaments of this size in mixed matrix conductors, development work is currently in progress at several government laboratories. The small size filaments can be produced by double stacking in the manufacture of a monolithic conductor, or by cabling a number of mixed matrix strands. It should be emphasized that the latter process is not necessary for the achievement of sufficiently low eddy current losses with mixed matrix strands, and in fact will only reduce eddy currents if the solder or insulation between strands is higher in resistivity than the effective transverse conductivity through the strands. In the long term, the manufacture of multithousand filament monoliths should be cheaper than cabling. 
VIII. Summary of Conductor Recommendations

- For a 30 second ramp, conductors 1 and 2 described in table 3 , which represent the lowest cost conductor designs, will meet the energy doubler requirements.

- A calculation of the average losses for conductors 1 and 2 is recommended to determine their applicability for a 10 second ramp.

- Cabled conductors $7,8,11$ and 12 represent state of the art cabled conductor designs which meet the energy doubler requirements for a 10 second ramp.

- A calculation of average losses for conductors 11 and 12 is recommended to determine their applicability for a four second ramp time.

- Monolithic mixed matrix conductors such as conductors 3 and 4, but containing $\sim 10 \mu$ copper clad filaments in a copper $10 \% \mathrm{~N}$ matrix are recommended for NAL development to meet the energy doubler requirements for a 4 second ramp time. 


\section{$\underline{\text { References }}$}

1. W.J. Carr, Jr., "AC Loss in a Twisted Filamentary Superconducting Wire," J. App1. Phys. 45, 929-34, February 1974.

2. W.J. Carr, Jr., "AC Loss in a Twisted Filamentary Superconducting Wire II," App1. Phys. 45, 935-38, February 1974.

3. W.J. Carr, Jr., "Conductivity, Permeability and Dielectric Constant in a Multifilament Superconductor," submitted to J. Appl. Phys.

(3) Paper 74-9C3-MACON-P1.

4. M.S. Walker and J.H. Murphy, Semiannual Report on ONR Contract \#N00014-73-C-0461.

5. W.J. Carr, Jr., "Electromagnetic Theory for llamentary Superconductors," submitted to Physical Review, Paper 74-9J2-MACON-P2.

6. W.J. Carr, Jr., M.S. Walker and J.H. Murphy, "Alternating Field Loss in a Multifilament Superconducting Wire for Weak A.C. Fields Superposed on a Contact Bias," submitted to J. App1. Phys., (-) Paper 74-9C3-MACON-P2

7. J.H. Murphy, "The Effect of DC Transport Current in Superconductors," Paper in preparation for ONR Contract N00014-73-C-0461.

8. R. Hancox, "Stability of Flux Motion in Superconducting Cylinders and Coils," Proc. 10th Intern. Conf. on Low Temperature Physics (1966).

9. J.H. Murphy, M.S. Walker, W.J. Carr, Jr., "Alternating Field Losses In a Rectangular Multifilamentary NbT1 Superconductor," Paper S-2 presented at the Applied Superconductivity Conference (1974). 
10. J.L. Kirtley, Jr., "Basic Formulas from Air-Core Synchronous Machines," IEEE Conference Paper 71CP155-PWR (1971).

11. R.A. Popley, D.J. Sambrook, C.R. Walters and M.N. Wilson, "A New Superconducting Composite with Low Hysteresis Loss," Proc. of the 1972 Applied Superconductivity Conf., p. 516-7 (1972). 